\title{
Control design with guaranteed ultimate bound for perturbed systems
}

\author{
Ernesto Kofman ${ }^{\mathrm{a}, 1}$ María M. Seron ${ }^{\mathrm{b}}$ Hernan Haimovich ${ }^{\mathrm{a}}$ \\ ${ }^{a}$ CONICET; Departamento de Electrónica, Facultad de Cs. Ex., Ing. y Agrim., Universidad Nacional de Rosario, Riobamba \\ 245bis, 2000 Rosario, Argentina \\ ${ }^{\mathrm{b}}$ Centre for Complex Dynamic Systems and Control (CDSC), School of Electrical Engineering and Computer Science, The \\ University of Newcastle, Callaghan, NSW 2308, Australia
}

\begin{abstract}
We present a new control design method for perturbed multiple-input systems, which guarantees any desired componentwise ultimate bound on the system state. The method involves eigenvalue/eigenvector assignment by state feedback and utilises a componentwise bound computation procedure. This procedure directly takes into account both the system and perturbation structures by performing componentwise analysis, thus avoiding the need for bounds on the norm of the perturbation. The perturbation description adopted can accommodate for numerous types of uncertainties, including uncertain time-delays in the feedback loop. We apply the method to an example taken from the literature to illustrate its simplicity and generality.
\end{abstract}

Key words: Ultimate bounds, robust control, uncertain systems, time delayed systems, eigenstructure assignment.

\section{Introduction}

The design of any realistic control system must necessarily take account of the effect of perturbations on the closed-loop system performance. Perturbations may arise from unknown disturbance signals, model uncertainty, unknown time delays, component ageing, etc. Typically, the exact value of a perturbation variable is unknown but assumed to be bounded. In the presence of bounded perturbations that do not vanish as the state approaches an equilibrium point, asymptotic stability is in general not possible. However, under certain conditions, the ultimate boundedness of the system's trajectories can be achieved (Brockman and Corless, 1998; Khalil, 2002). A guaranteed ultimate bound on the system's trajectories can be associated with good "attenuation" of the effect of perturbations.

A standard approach for the computation of ultimate bounds is the use of level sets of suitable Lyapunov functions [see, for example, Khalil (2002, Section 9.2)]. The Lyapunov approach is a powerful tool that can be applied to very general nonlinear systems. However, this approach may result in conservative bounds in the linear case due to the loss of the structure of the system and

\footnotetext{
1 Corresponding author. Email: kofman@fceia.unr.edu.ar
}

also possibly of the perturbation, whose norm typically needs to be bounded for the analysis. Kofman (2005), Kofman et al. (2007a) and Haimovich (2006) presented a new method for ultimate bound computation based on componentwise analysis of the system in modal coordinates. This method directly derives componentwise ultimate bounds, exploiting the system geometry as well as the perturbation structure without requiring either the computation of a Lyapunov function for the system or bounding the norm of the perturbation vector. The examples in Kofman (2005), Kofman et al. (2007a) and Haimovich (2006) show that this componentwise approach may provide bounds that are much tighter than those obtained via standard Lyapunov analysis.

When perturbations span the same subspace spanned by the control input, commonly referred to as matched perturbations, then an ultimate bound on the closed-loop trajectories can be arbitrarily assigned (Schmitendorf and Barmish, 1986). Several control design methods that can achieve an arbitrarily small ultimate bound for linear uncertain systems have been reported in the robust control literature (see, for example, Barmish et al., 1983; Trinh and Aldeen, 1996; Cao and Sun, 1998; Oucheriah, 1999). These methods are based on Lyapunov analysis.

This paper presents a novel controller design method for multiple-input perturbed linear continuous-time sys- 
tems where every component of the perturbation vector is bounded by a (possibly) delayed-state-dependent function. Given desired ultimate bounds for each component of the system state, a suitable state-feedback matrix can be systematically computed via this method so that the corresponding closed-loop system exhibits the desired componentwise ultimate bound. This controller design method employs the componentwise approach of Kofman (2005), Kofman et al. (2007a) and Haimovich (2006). In this context, the contribution of the current paper is threefold. First, we further extend the aforementioned componentwise approach to linear systems with perturbations bounded by a function of previous values of the state. Second, we provide a sufficient condition for a perturbed system to be ultimately bounded when the perturbation is componentwise bounded as explained. Third and most importantly, we develop the aforementioned systematic controller design method. An important property of the ultimate bounds considered throughout this paper is that they are globally valid, i.e., they hold irrespective of the initial condition of the system, as opposed to the local ultimate bounds in Kofman et al. (2007a). A preliminary version of this paper was presented in the conference paper Kofman et al. (2007b).

Notation. $\mathbb{Z}, \mathbb{R}$ and $\mathbb{C}$ denote the sets of integer, real and complex numbers. $|M|$ and $\mathbb{R e}(M)$ denote the elementwise magnitude and real part, respectively, of a (possibly complex) matrix or vector $M$. Also, $\boldsymbol{\rho}(M)$ denotes the spectral radius of the matrix $M$. If $x(t)$ is a vector-valued function, then $\lim \sup _{t \rightarrow \infty} x(t)$ denotes the vector obtained by taking limsup $\operatorname{su}_{t \rightarrow \infty}$ of each component of $x(t)$. Similarly, 'lim' and 'max' denote componentwise operations on a vector. The expression $x \preceq y(x \prec y)$ denotes the set of componentwise inequalities $x_{i} \leq y_{i}\left(x_{i}<y_{i}\right)$ between the elements of the real vectors $x$ and $y$, and similarly for $x \succeq y(x \succ y)$ and in the case when $x$ and $y$ are matrices. $\mathbb{R}_{+}$and $\mathbb{R}_{+, 0}$ denote the positive and nonnegative real numbers, respectively, and similarly for $\mathbb{Z}_{+}$ and $\mathbb{Z}_{+, 0}$. For $c \in \mathbb{C}, \bar{c}$ denotes its complex conjugate.

\section{Problem formulation}

Consider the multiple-input linear perturbed system

$$
\dot{x}(t)=A x(t)+B u(t)+H w(t),
$$

where $x(t) \in \mathbb{R}^{n}$ is the system state, $u(t) \in \mathbb{R}^{m}$ is the control input, $A \in \mathbb{R}^{n \times n}, B \in \mathbb{R}^{n \times m}, H \in \mathbb{R}^{n \times k}$ are constant matrices, $(A, B)$ is a controllable pair, $B$ has full column rank, and the perturbation variable $w(t) \in \mathbb{R}^{k}$ satisfies the componentwise bound

$$
|w(t)| \preceq F \theta(t)+\bar{w} \text { for all } t \geq 0,
$$

with $F \in \mathbb{R}_{+, 0}^{k \times n}, \bar{w} \in \mathbb{R}_{+, 0}^{k}$, and $\theta(t) \in \mathbb{R}_{+, 0}^{n}$ defined as

$$
\theta(t) \triangleq \max _{t-\bar{\tau} \leq \tau \leq t}|x(\tau)|
$$

where $\bar{\tau} \geq 0$ and the maximum is taken componentwise. We emphasize that (2) expresses a bound for each one of the $k$ components of the perturbation vector $w(t)$.

Given an arbitrary positive vector $b^{\star} \in \mathbb{R}_{+}^{n}$, our goal is to design a linear state-feedback control $u(t)=K x(t), K \in$ $\mathbb{R}^{m \times n}$, such that the solutions to $\dot{x}(t)=(A+B K) x(t)+$ $H w(t)$, where $w(t)$ is componentwise bounded as in (2)(3), are ultimately bounded with componentwise ultimate bound $b^{\star}$, that is, they satisfy

$$
\limsup _{t \rightarrow \infty}|x(t)| \preceq b^{\star} .
$$

When the perturbation bounds are constant, i.e., when $F=0$ in (2), and provided the perturbations are matched, i.e., if $H=B G$ for some $G \in \mathbb{R}^{m \times k}$, it is wellknown that the above goal is achievable (Schmitendorf and Barmish, 1986). However, additional assumptions must be made when the perturbation bound depends on either the current or a delayed version of the system state, as when $F \neq 0$ in (2). In the current paper, we will provide a sufficient condition for achieving any desired componentwise ultimate bound and a method for computing the corresponding feedback matrix $K$.

Remark 2.1 The setting (1)-(3) describes, inter-alia, any combination of the following types of uncertainty:

- Uncertainty in the system evolution matrix, where $\dot{x}(t)=(A+\Delta A(t)) x(t)+B u(t)$, and $|\Delta A(t)| \preceq \overline{\Delta A}$, for all $t \geq 0$; in this case, we can take $H=\mathrm{I}$ in (1), $F=\overline{\Delta A}$ and $\bar{w}=0$ in (2), and $\bar{\tau}=0$ in (3).

- Uncertainty in the system input matrix lassuming that $u(t)=K x(t)$ in (1)], where $\dot{x}(t)=A x(t)+(B+$ $\Delta B(t)) K x(t)$, and $|\Delta B(t)| \preceq \overline{\Delta B}$, for all $t \geq 0$; in this case, we can take $H=\mathrm{I}$ in (1), $F=\overline{\Delta B}|K|$ and $\bar{w}=0$ in (2), and $\bar{\tau}=0$ in (3).

- Uncertain time delay, where $w(t)=A_{d} x(t-\tau)$, and $0 \leq \tau \leq \tau_{\max }$; in this case, we can take $F=\left|A_{d}\right|$ and $\bar{w}=0$ in (2), and $\bar{\tau}=\tau_{\max }$ in (3).

- Disturbances with constant bounds, i.e., $F=0$ in (2).

\section{Componentwise ultimate bounds under per- turbations}

When a state-feedback control $u(t)=K x(t)$ is applied to system (1), the resulting closed-loop system takes the form

$$
\dot{x}(t)=\bar{A} x(t)+H w(t),
$$

where $\bar{A}=A+B K$. The following theorem provides a closed-form expression for an ultimate bound of system (5) when the perturbation $w(t)$ is bounded as in $(2)-(3)$.

Theorem 3.1 Consider system (5) where $x(t) \in \mathbb{R}^{n}$, $w(t) \in \mathbb{R}^{k}, \bar{A} \in \mathbb{R}^{n \times n}$ and $H \in \mathbb{R}^{n \times k}$. Let $\bar{A}$ be a Hurwitz matrix with Jordan canonical form $\Lambda=V^{-1} \bar{A} V$. 
Suppose that (2) and (3) hold with $F \in \mathbb{R}_{+, 0}^{k \times n}, \bar{w} \in \mathbb{R}_{+, 0}^{k}$ and $\bar{\tau} \in \mathbb{R}_{+, 0}$. Define the matrix

$$
R \triangleq|V|\left|[\mathbb{R e}(\Lambda)]^{-1}\right|\left|V^{-1} H\right|
$$

suppose that $\boldsymbol{\rho}(R F)<1$ and let $b \in \mathbb{R}_{+, 0}^{n}$ be given by ${ }^{2}$

$$
b \triangleq(\mathrm{I}-R F)^{-1} R \bar{w} .
$$

Then, $\lim \sup _{t \rightarrow \infty}|x(t)| \preceq b$.

PROOF. Consider the map $T: \mathbb{R}_{+, 0}^{n} \rightarrow \mathbb{R}_{+, 0}^{n}$ defined by $T(x) \triangleq R(F x+\bar{w})$. For every $\gamma \in \mathbb{R}_{+, 0}^{n}$ and $\ell \in \mathbb{Z}_{+, 0}$ consider the map $T_{\gamma}^{\ell}: \mathbb{R}_{+, 0}^{n} \rightarrow \mathbb{R}_{+, 0}^{n}$ defined by

$$
T_{\gamma}^{0}(x) \triangleq T(x)+|V| \gamma, \quad T_{\gamma}^{\ell+1}(x) \triangleq T_{\gamma}^{0}\left(T_{\gamma}^{\ell}(x)\right) .
$$

The proof requires the following claims, whose proofs are given in Appendix A.

Claim 1 There exists $\bar{x} \in \mathbb{R}_{+, 0}^{n}$, which may depend on $\theta(0)$ [see (3)], such that $|x(t)| \preceq \bar{x}$ for all $t \geq-\bar{\tau}$.

Claim 2 Suppose that $|x(t)| \preceq \bar{x}$ for all $t \geq-\bar{\tau}$. Then, for any $\gamma \in \mathbb{R}_{+}^{n}$ and $\ell \in \mathbb{Z}_{+, 0}$, there exists a time instant $\bar{t}=\bar{t}(\ell, \gamma) \geq 0$ such that $|x(t)| \preceq T_{\gamma}^{\ell+1}(\bar{x})$, for all $t \geq \bar{t}$.

Claim 3 Suppose that $|x(t)| \preceq \bar{x}$ for all $t \geq-\bar{\tau}$. Then, for any $\epsilon \in \mathbb{R}_{+}^{n}$, there exist $\ell=\ell(\epsilon) \in \mathbb{Z}_{+, 0}$ and $\gamma=$ $\gamma(\epsilon) \in \mathbb{R}_{+}^{n}$ such that $T_{\gamma}^{\ell+1}(\bar{x}) \prec b+\epsilon$.

Claim 1 shows that for every initial condition $\theta(0)$, $x(t)$ remains bounded with a bound that may depend on the initial condition $\theta(0)$. Claims 2 and 3 combined show that, provided $x(t)$ remains bounded, $x(t)$ can be bounded componentwise with a bound that becomes arbitrarily close to $b$ as $t \rightarrow \infty$, and that this holds irrespective of the initial condition. These facts imply the result, namely that $\lim \sup _{t \rightarrow \infty}|x(t)| \preceq b$. We next rigorously establish the result. From Claims 1 and 3, it straightforwardly follows that for any $\epsilon \in \mathbb{R}_{+}^{n}$, there exist $\ell=\ell(\epsilon) \in \mathbb{Z}_{+, 0}$ and $\gamma=\gamma(\epsilon) \in \mathbb{R}_{+}^{n}$ such that $T_{\gamma}^{\ell+1}(\bar{x}) \prec b+\epsilon$. For these values of $\ell$ and $\gamma$, we have, from Claim 2, that there exists $\bar{t}=\bar{t}(\ell, \gamma) \geq 0$ such that $|x(t)| \preceq T_{\gamma}^{\ell+1}(\bar{x}) \prec b+\epsilon$, for all $t \geq \bar{t}$. This implies that $\lim \sup _{t \rightarrow \infty}|x(t)| \preceq b$, concluding the proof.

Theorem 3.1 provides the first two contributions of the paper. First, it extends the componentwise framework of Kofman (2005), Kofman et al. (2007a) and Haimovich (2006) to the case when the perturbation bound may depend on previous values of the state. In this context, we stress that the ultimate bound $b$ in (7) is a vector

\footnotetext{
2 Since $\rho(R F)<1$, then the matrix I $-R F$ is invertible (see, for example, Horn and Johnson, 1985).
}

having $n$ components, each of which constitutes an ultimate bound for the corresponding component of the state $x$. As a second contribution, it provides a sufficient condition, namely that $\bar{A}$ be Hurwitz and $\rho(R F)<1$, for system (5) to be ultimately bounded when the perturbation is bounded as in (2)-(3).

\section{Control design with guaranteed component- wise ultimate bound}

The componentwise ultimate bound $b$ provided by Theorem 3.1 in (7) depends, through the matrix $R$ defined in (6), on the eigenstructure matrices $\Lambda$ and $V$ of the closed-loop system matrix $\bar{A}=V \Lambda V^{-1}=A+B K$ which, in turn, depends on the state-feedback matrix $K$. In this section, we exploit the dependency of $b$ on $\Lambda$ and $V$ jointly with the dependency of these matrices on $K$. This allows us to develop a new control design procedure to guarantee any desired componentwise ultimate bound for systems with matched perturbations.

In essence, our derivations below are as follows. Given a desired componentwise ultimate bound $b^{\star} \in \mathbb{R}_{+}^{n}$, we will select a suitable eigenstructure for the controller canonical form of the matrix $\bar{A}$ so that the desired ultimate bound is achieved. Once this eigenstructure is selected, we will compute the required state-feedback matrix $K$. These steps will be performed by means of techniques of eigenvalue/eigenvector assignment by state feedback (Sinswat and Fallside, 1977), as we show below.

We consider the perturbed multiple-input system (1)(3) and let $x=U x_{c}$ be the transformation that brings system (1) into its controller canonical form (see, for example, Luenberger (1967)):

$$
\dot{x}_{c}(t)=A_{c} x_{c}(t)+B_{c} u(t)+U^{-1} H w(t),
$$

with $A_{c}=U^{-1} A U$ and $B_{c}=U^{-1} B$. Define

$$
\theta_{c}(t) \triangleq \max _{t-\bar{\tau} \leq \tau \leq t}\left|x_{c}(t)\right|
$$

and let $w(t)$ be bounded as follows [c.f. (2), (3) and (10)]:

$$
|w(t)| \preceq F|U| \theta_{c}(t)+\bar{w} .
$$

The following lemma, whose proof is straightforward, shows how finding an ultimate bound for the transformed system (9) gives a bound on the system in the original coordinates.

Lemma 4.1 Consider system (9)-(11) and suppose that $\limsup _{t \rightarrow \infty}\left|x_{c}(t)\right| \preceq b_{c}$ when a state-feedback control $u(t)=K_{c} x_{c}(t)$ is applied. Then, $\lim \sup _{t \rightarrow \infty}|x(t)| \preceq$ $|U| b_{c}$, where $x(t)$ is the state of system (1)-(3) with the feedback control $u(t)=K_{c} U^{-1} x(t)$. 
The following theorem shows, as an intermediate tool, how we may select eigenvalues and eigenvectors for the closed-loop system in controller canonical form and how we may compute the corresponding feedback matrix $K_{c}$. The proof is given in Appendix B.

Theorem 4.2 Select an eigenvalue matrix $\Lambda=$ $\operatorname{diag}\left(\lambda_{1}, \ldots, \lambda_{n}\right)$, where $\lambda_{i} \in \mathbb{C}, i=1, \ldots, n$, satisfy $\lambda_{i} \neq \lambda_{j}$ whenever $i \neq j, \mathbb{R e}\left(\lambda_{i}\right)<0$, and if $\lambda_{i} \notin \mathbb{R}$, then either $\lambda_{i-1}=\overline{\lambda_{i}}$ or $\lambda_{i+1}=\overline{\lambda_{i}}$. Let $d_{i}, i=1, \ldots, m$, be the controllability indices of the system ${ }^{3}$ and select numbers $e_{i, j} \in \mathbb{C}, i=1, \ldots, m, j=1, \ldots, n$ so that $e_{i, j+1}=\overline{e_{i, j}}$ whenever $\lambda_{j+1}=\overline{\lambda_{j}}$ and such that the matrix

$$
V \triangleq\left[\begin{array}{ccc}
V_{1,1} & \ldots & V_{1, n} \\
\vdots & \ddots & \vdots \\
V_{m, 1} & \cdots & V_{m, n}
\end{array}\right], V_{i, j} \triangleq\left[\begin{array}{c}
e_{i, j} \lambda_{j}^{-\left(d_{i}-1\right)} \\
\vdots \\
e_{i, j} \lambda_{j}^{-1} \\
e_{i, j}
\end{array}\right]
$$

has linearly independent columns. Let $A_{c}, B_{c}$ be the matrices of system (9) in controller canonical form and let

$$
K_{c}=B_{c}^{\dagger}\left(V \Lambda V^{-1}-A_{c}\right),
$$

where $B_{c}^{\dagger} \triangleq\left(B_{c}^{T} B_{c}\right)^{-1} B_{c}^{T}$. Then, $A_{c}+B_{c} K_{c}=V \Lambda V^{-1}$.

We next show that for any initially chosen eigenvalue and eigenvector matrices $\Lambda$ and $V$ as in Theorem 4.2, a new set of eigenvalues consisting of a scaled version of the original ones leads to a feedback gain that achieves the desired ultimate bound $b^{\star}$ for system (1)-(3).

Theorem 4.3 Consider the perturbed system (1)-(3), where $(A, B)$ is a controllable pair and $H=B G$ for some $G \in \mathbb{R}^{m \times k}$. For each $\mu>0$ consider the scaled eigenvalue matrix $\Lambda=\operatorname{diag}\left(\lambda_{1}, \ldots, \lambda_{n}\right)$, where $\lambda_{i}=\mu \tilde{\lambda}_{i}$ for $i=1, \ldots, n$ and $\tilde{\lambda}_{i} \in \mathbb{C}, i=1, \ldots, n$, satisfy $\tilde{\lambda}_{i} \neq \tilde{\lambda}_{j}$ whenever $i \neq j, \mathbb{R e}\left(\tilde{\lambda}_{i}\right)<0$, and if $\tilde{\lambda}_{i} \notin \mathbb{R}$, then either $\tilde{\lambda}_{i-1}=\overline{\tilde{\lambda}}_{i}$ or $\tilde{\lambda}_{i+1}=\overline{\tilde{\lambda}}_{i}$. Select complex numbers $e_{i, j}$, $i=1, \ldots, m, j=1, \ldots, n$ as explained in Theorem 4.2 and consider the corresponding matrix $V$ in (12). Let $x=U x_{c}$ be the state variable transformation that brings system (1) into its controller canonical form (9) with matrices $A_{c}=U^{-1} A U$ and $B_{c}=U^{-1} B$, and define

$$
R_{\mu} \triangleq|V|\left|[\mathbb{R e}(\Lambda)]^{-1}\right|\left|V^{-1} U^{-1} H\right|
$$

where the subscript $\mu$ is added to stress the dependency of $R_{\mu}$ on the scaling factor $\mu$. Then:

a) If $\mu$ is such that $\boldsymbol{\rho}\left(R_{\mu} F|U|\right)<1$, then the state of the closed-loop system (1)-(3) under the state feedback

\footnotetext{
3 The controllability indices satisfy $d_{i} \geq 1$, for $i=1, \ldots, m$ and $\sum_{i=1}^{m} d_{i}=n$, see for example, Wolovich (1974).
}

$u(t)=K x(t)$, with $K=K_{c} U^{-1}$ and $K_{c}$ as in (13), satisfies $\lim \sup _{t \rightarrow \infty}|x(t)| \preceq b_{\mu}$, where

$$
b_{\mu} \triangleq|U|\left(\mathrm{I}-R_{\mu} F|U|\right)^{-1} R_{\mu} \bar{w} .
$$

b) The product $\mu \rho\left(R_{\mu} F|U|\right)$ is a nonincreasing function of $\mu$. Hence, there exists $\bar{\mu} \in \mathbb{R}_{+}$such that $\rho\left(R_{\mu} F|U|\right)<1$ for all $\mu>\bar{\mu}$.

c) For $\mu>\bar{\mu}$, the product $\mu b_{\mu}$, with $b_{\mu}$ calculated in (15), is a componentwise nonincreasing function of $\mu$. Hence, for $\mu>\bar{\mu}$, the ultimate bound $b_{\mu}$ in (15) satisfies $\lim _{\mu \rightarrow \infty} b_{\mu}=0$.

PROOF. a) Note that $A_{c}+B_{c} K_{c}$ is Hurwitz because its eigenvalues, the nonzero entries of $\Lambda$, all have negative real part. Hence, applying Theorem 3.1 to system (9)-(11), it follows that $\limsup _{t \rightarrow \infty}\left|x_{c}(t)\right| \preceq(\mathrm{I}-$ $\left.R_{\mu} F|U|\right)^{-1} R_{\mu} \bar{w}$, provided $\rho\left(R_{\mu} F|U|\right)<1$. The result then follows by application of Lemma 4.1.

b) From the forms of $\Lambda=\operatorname{diag}\left(\lambda_{1}, \ldots, \lambda_{n}\right)$ with $\lambda_{i}=\mu \tilde{\lambda}_{i}$ for $i=1, \ldots, n$, and of $V$ in (12), we can write

$$
\begin{gathered}
\Lambda=\mu \tilde{\Lambda}, \quad V=\mathcal{R}(\mu) \mathcal{E}, \quad \text { where } \\
\tilde{\Lambda} \triangleq \operatorname{diag}\left(\tilde{\lambda}_{1}, \ldots, \tilde{\lambda}_{n}\right), \\
\mathcal{R}(\mu) \triangleq \operatorname{diag}\left(\mu^{-\left(d_{1}-1\right)}, \ldots, \mu^{-1}, 1, \mu^{-\left(d_{2}-1\right)}, \ldots, \mu^{-1}, 1\right. \\
\left.\ldots, \mu^{-\left(d_{m}-1\right)}, \ldots, \mu^{-1}, 1\right) .
\end{gathered}
$$

and $\mathcal{E}$ is an invertible matrix independent of $\mu$. From (16), it follows that $[\operatorname{Re}(\Lambda)]^{-1}=\mu^{-1}[\operatorname{Re}(\tilde{\Lambda})]^{-1}$. Since $H=B G$ and using $B_{c}=U^{-1} B$ we have that $U^{-1} H=U^{-1} B G=B_{c} G$. Since the system is in controller canonical form, the specific form of the matrix $B_{c}$ is such that $\mathcal{R}^{-1}(\mu) B_{c}=B_{c}$, and hence $V^{-1} U^{-1} H=\mathcal{E}^{-1} \mathcal{R}^{-1}(\mu) B_{c} G=\mathcal{E}^{-1} B_{c} G$. Using these facts, the matrix $R_{\mu}$ in (14) takes the form

$$
\begin{aligned}
R_{\mu} & =|V| \cdot\left|\mu^{-1}[\operatorname{Re}(\tilde{\Lambda})]^{-1}\right|\left|\mathcal{E}^{-1} B_{c} G\right| \\
& =\mu^{-1} \mathcal{R}(\mu)|\mathcal{E}| \cdot\left|[\operatorname{Re}(\tilde{\Lambda})]^{-1}\right|\left|\mathcal{E}^{-1} B_{c} G\right|,
\end{aligned}
$$

where the last line follows from (16) and the facts that $\mu>0$ and $\mathcal{R}(\mu)$ is diagonal with positive diagonal entries. Defining $\bar{R} \triangleq|\mathcal{E}| \cdot\left|\mathbb{R e}(\tilde{\Lambda})^{-1}\right|\left|\mathcal{E}^{-1} B_{c} G\right|$, with rows $\bar{R}_{1}, \bar{R}_{2}, \ldots, \bar{R}_{n}$, we have from (19) and (18) that

$$
R_{\mu}=\left[\begin{array}{c}
R_{\mu}^{1} \\
\vdots \\
R_{\mu}^{m}
\end{array}\right], \quad R_{\mu}^{i}=\left[\begin{array}{c}
\mu^{-d_{i}} \bar{R}_{\sigma_{i}-d_{i}+1} \\
\mu^{-d_{i}+1} \bar{R}_{\sigma_{i}-d_{i}+2} \\
\vdots \\
\mu^{-1} \bar{R}_{\sigma_{i}}
\end{array}\right]
$$

where $\sigma_{i} \triangleq \sum_{j=1}^{i} d_{j}$ for $i=1, \ldots, m$. Since $\bar{R}$ is independent of $\mu$ and has nonnegative entries, then all en- 
tries of the product $\mu R_{\mu}$ are nonincreasing with $\mu$. Then, $\boldsymbol{\rho}\left(\mu R_{\mu} F|U|\right)=\mu \boldsymbol{\rho}\left(R_{\mu} F|U|\right)$ is nonincreasing with $\mu$. This establishes part $\mathrm{b})$.

c) Define the function $b_{c}(\mu) \triangleq\left(\mathrm{I}-R_{\mu} F|U|\right)^{-1} R_{\mu} \bar{w}$. Notice that $b_{c}(\mu)$ satisfies

$$
b_{c}(\mu)=R_{\mu} F|U| b_{c}(\mu)+R_{\mu} \bar{w} .
$$

Differentiating (21) with respect to $\mu$ we obtain $\frac{d b_{c}}{d \mu}(\mu)=$ $D_{\mu} F|U| b_{c}(\mu)+R_{\mu} F|U| \frac{d b_{c}}{d \mu}(\mu)+D_{\mu} \bar{w}$, where $D_{\mu} \triangleq \frac{d R_{\mu}}{d \mu}$. Then, $\frac{d b_{c}}{d \mu}(\mu)=\left(\mathrm{I}-R_{\mu} F|U|\right)^{-1} D_{\mu}\left[F|U| b_{c}(\mu)+\bar{w}\right]$. Since $\boldsymbol{\rho}\left(R_{\mu} F|U|\right)<1$ for all $\mu>\bar{\mu}$, and $R_{\mu} F|U| \succeq 0$, then $\left(\mathrm{I}-R_{\mu} F|U|\right)^{-1} \succeq 0$. Also, note that $\left[F|U| b_{c}(\mu)+\bar{w}\right] \succeq 0$ for all $\mu>\bar{\mu}$. From (20), note that all the entries of $D_{\mu} \triangleq$ $\frac{d R_{\mu}}{d \mu}$ are nonpositive. Therefore, all the entries of $\frac{d b_{c}}{d \mu}$ are nonpositive and it follows that $b_{c}(\mu)$ is nonincreasing. Since $b_{\mu}$ in (15) satisfies $b_{\mu}=|U| b_{c}(\mu)$, it follows from (21) that $\mu b_{\mu}=|U| \mu R_{\mu}\left[F|U| b_{c}(\mu)+\bar{w}\right]$. The terms $|U|$, $\mu R_{\mu}$-from the proof of part b)- and $\left[F|U| b_{c}(\mu)+\bar{w}\right]$ are non increasing with $\mu$. Then, $\mu b_{\mu}$ is nonincreasing, which concludes the proof.

Part a) of Theorem 4.3 gives an ultimate bound expression for system (1)-(3) jointly with the corresponding feedback matrix for different values of the scaling factor $\mu$. Parts b) and c) establish properties that the different quantities involved have as functions of $\mu$. These properties are used in Algorithm 4.4 below to develop a systematic design procedure to compute a suitable feedback matrix that achieves any desired ultimate bound.

\section{Algorithm 4.4 (Systematic Design Procedure)} Given a desired componentwise ultimate bound $b^{\star}$ :

(1) Compute the matrix U that brings system (1) into its controller canonical form (9), and the transformed matrices $A_{c} \triangleq U^{-1} A U$ and $B_{c} \triangleq U^{-1} B$.

(2) Choose an initial configuration $\left\{\tilde{\lambda}_{i}\right\}_{i=1}^{n}$ of $n$ distinct and stable eigenvalues, where complex eigenvalues appear in complex conjugate pairs.

(3) Find the controllability indices $d_{i}, i=1, \ldots, m$, of system (1) and define $\sigma_{i} \triangleq \sum_{j=1}^{i} d_{j}$ for $i=$ $1, \ldots, m$. Select $e_{i, j}, i=1, \ldots, m, j=1, \ldots, n$ as required in Theorem $4.2^{4}$.

(4) Select a scaling factor $\mu>0\left(\mu \in \mathbb{R}_{+}\right)$.

(5) Compute $V$ in (12) using the values of $e_{i, j}, i=$ $1, \ldots, m, j=1, \ldots, n$, chosen in step 3 and $\lambda_{j}=$ $\mu \tilde{\lambda}_{j}$ for $j=1, \ldots, n$. Set $\Lambda \triangleq \operatorname{diag}\left(\lambda_{1}, \ldots, \lambda_{n}\right)$.

\footnotetext{
$\overline{4}$ It is easy to check that selecting $e_{i, j}=\tilde{\lambda}_{j}^{\sigma_{i}-n}$, for example, makes the columns of $V$ in (12) linearly independent for all values of $\mu>0$ and is such that $e_{i, j+1}=\overline{e_{i, j}}$ whenever $\lambda_{j+1}=\overline{\lambda_{j}}$, as required in Theorem 4.2.
}

(6) Compute $R_{\mu}$ in (14) and check the condition $\boldsymbol{\rho}\left(R_{\mu} F|U|\right)<1$. If not satisfied, increase $\mu$ and go to step 5. According to Part b) of Theorem 4.3 any new value $\tilde{\mu}>\mu \rho\left(R_{\mu} F|U|\right)$ ensures that $\boldsymbol{\rho}\left(R_{\tilde{\mu}} F|U|\right)<1$.

(7) Compute $b_{\mu}$ in (15) and check the condition $b_{\mu} \preceq b^{\star}$. If not satisfied, increase $\mu$ and go to step 5. According to Part $c$ ) of Theorem 4.3 the new value $\tilde{\mu}=\mu \max _{i}\left(b_{\mu_{i}} / b_{i}^{\star}\right)$ ensures that $b_{\tilde{\mu}} \preceq b^{\star}$.

(8) Compute $V$ as in (12) and $K=K_{c} U^{-1}$ with $K_{c}$ as in (13). Applying the feedback control $u(t)=K x(t)$ achieves the desired ultimate bound. In other words, $\lim \sup _{t \rightarrow \infty}|x(t)| \preceq b^{\star}$.

Note that if one needs to increase $\mu$ in step (6) [and similarly in step (7)], the suggested new value $\tilde{\mu}$ causes the algorithm to return to step (5) at most once.

\section{Example}

We consider the rotational motion of a rigid spacecraft subject to bounded disturbances (Brockman and Corless, 1998). Under the assumption that the spacecraft is axisymmetric about the third body-fixed axis and there are no torques about the symmetry axis, the system can be described by $(1)$, where $x(t) \in \mathbb{R}^{2}$ is formed by the components of the spacecraft inertial angular velocity with respect to the first two axes and $u(t) \in \mathbb{R}^{2}$, $w(t) \in \mathbb{R}^{2}$ are, respectively, control input and unknown bounded disturbance torques about the first two axes. The matrices in (1) are $A=\left[\begin{array}{cc}0 & -0.05 \\ 0.05 & 0\end{array}\right], B=H=$ $0.01 \mathrm{I}_{2}$, where $\mathrm{I}_{2}$ is the $2 \times 2$ identity matrix. The disturbance bounds are $|w(t)| \preceq \bar{w}=\left[\begin{array}{ll}1 & 1\end{array}\right]^{T} / \sqrt{2}$ for all $t \geq 0$.

Our first goal is to use Algorithm 4.4 to design a state feedback gain such that a componentwise ultimate bound $b^{\star}=\left[\begin{array}{ll}0.01 & 0.01\end{array}\right]^{T}$ can be guaranteed for the system state. Step (1): the matrix $U=0.01 \mathrm{I}_{2}$ transforms the system into controller canonical form. Step (2): we choose $\tilde{\lambda}_{1}=(-1+j) / \sqrt{2}$ and $\tilde{\lambda}_{2}=\overline{\tilde{\lambda}_{1}}$. Steps (3)-(6): we select $\mu=1$ and compute $V$ and $\Lambda$ as indicated (to compute $V$ we use the choice of $e_{i, j}$ described in foonote 4$)$; note that the condition $\boldsymbol{\rho}\left(R_{\mu} F|U|\right)<1$ is trivially satisfied since $F=0$. Step (7): from (15) compute $b_{\mu}=\left[\begin{array}{ll}0.0283 & 0.0283\end{array}\right]^{T}$; since $b_{\mu} \succeq b^{\star}$ take $\tilde{\mu}=\mu 0.0283 / 0.01$. We obtain $b_{\tilde{\mu}}=\left[\begin{array}{ll}0.01 & 0.01\end{array}\right]^{T} \preceq b^{\star}$ as desired. Step (8): the corresponding gain is $\tilde{\tilde{K}}=\left[\begin{array}{cc}0 & 287.843 \\ -287.843 & -400\end{array}\right]$.

Our second goal is to exploit the degree of freedom provided by different choices of the eigenvector matrix $V$ given in (12), so as to minimise the ultimate bound on the second state $x_{2}$. Towards this goal, we fix $\mu=\tilde{\mu}$ obtained in Step (7) above and change the parameters $e_{i, j}$ in $V$ to minimise the second component of $b_{\mu}$ given in (15), subject to the constraint that the ultimate bound on the first state $x_{1}$ is less than or equal to 0.5 . We use 
Matlab's routine fminsearch for the optimisation and obtain $V=\left[\begin{array}{l}-0.4703-j 1.4169-0.4703+j 1.4169 \\ -0.0101+j 0.0034-0.0101-j 0.0034\end{array}\right]$. Using this value of $V$ and $\mu=\tilde{\mu}$ in (15) gives $b_{\mu}=\left[\begin{array}{ll}0.5 & 0.0036\end{array}\right]^{T}$ The controller gain for this case, $K=K_{c} U^{-1}$ with $K_{c}$ as in (13), is $K=-\left[\begin{array}{cc}200.011 & 28079.24 \\ 3.576 & 199.989\end{array}\right]$.

For comparison, we will consider the closed-loop system with the above gain $K$ and compute ultimate bounds using the method proposed in Brockman and Corless (1998). This method provides an upper bound on the ultimate size of the 2 -norm of the state as $\|x(t)\| \leq r \triangleq$ $\left[\lambda_{\max }(S)\right]^{1 / 2}\left(\lambda_{\max }\right.$ denotes the largest eigenvalue of a matrix), where $S>0$ is a solution of the Lyapunov equation $(A+B K) S+S(A+B K)^{T}+\alpha S+\alpha^{-1} H H^{T}=0$ for $0<\alpha<\bar{\alpha}$, and where $-2 \bar{\alpha}$ denotes the maximum of the real parts of the eigenvalues of $A+B K$. Minimising $r$ for $\alpha$ in the above range yields $r=0.2775$. Comparing with $b_{\mu}=\left[\begin{array}{ll}0.5 & 0.0036\end{array}\right]^{T}$ obtained above with our method we note that the latter gives a bound 78 times tighter for the second component of the state while the bound on the norm of the state is less than 2 times worse than that provided by the method of Brockman and Corless (1998) (since $\left\|b_{\mu}\right\| \approx 0.5<2 r$ ). We note that the latter method can be used to compute ultimate bounds once a linear controller is given but it is not evident how the method can be employed in a controller design procedure to assign ultimate bounds. On the other hand, the method proposed here can be used to design a feedback gain to achieve any desired ultimate bound and, moreover, has the flexibility to focus on bounds for the individual state components, as demonstrated in this example.

\section{Conclusions}

We have presented a new control design method which can guarantee a prespecified ultimate bound on the closed-loop trajectories of perturbed multiple-input systems. The method employs techniques of eigenvalue/eigenvector assignment by state feedback and a componentwise bound computation procedure. The latter procedure exploits the system geometry as well as the perturbation structure without requiring Lyapunov analysis or bounds on the norm of the perturbation. The perturbation description adopted can accommodate for numerous types of uncertainties, including the case of uncertain time-delays in the feedback loop.

\section{A Proofs of Claims 1-3}

We require the following additional results (Claim 4 and Lemma A.1). The proof of Lemma A.1 combines minor modifications to the proofs of Theorem 3 of Kofman (2005), Theorem 1 of Kofman et al. (2007a), and Theorem 7.3 of Haimovich (2006), and is therefore omitted.

Claim 4 For any $\theta_{0}, \delta \in \mathbb{R}_{+, 0}^{n}$, there exists $\beta \succ \theta_{0}$ such that $R F \beta+\delta \prec \beta$.
PROOF. It follows from $R F \succeq 0, \boldsymbol{\rho}(R F)<1$, and $\theta_{0} \succeq$ 0 (see for example, Horn and Johnson, 1985, §8).

Lemma A.1 Consider the system

$$
\dot{y}(t)=\bar{A}\left(y(t)+\xi_{0}\right)+H w(t),
$$

where $y(t), \xi_{0} \in \mathbb{R}^{n}, w(t) \in \mathbb{R}^{k}, \bar{A} \in \mathbb{R}^{n \times n}$ and $H \in$ $\mathbb{R}^{n \times k}$. Let $\vec{A}$ be Hurwitz with Jordan canonical form $\Lambda=$ $V^{-1} \bar{A} V$, let $\tilde{R} \triangleq|V|\left|[\mathbb{R e}(\Lambda)]^{-1}\right|\left|V^{-1} \bar{A}\right|$ and consider the matrix $R$ defined in (6). Let $w_{m} \in \mathbb{R}_{+, 0}^{k}$ and $t_{c} \in \mathbb{R}_{+, 0}$.

a) Suppose that $|w(t)| \preceq w_{m}$ for $0 \leq t \leq t_{c}$ and $y(0)=0$. Then, $|y(t)| \preceq R w_{m}+\tilde{R}\left|\xi_{0}\right|$ for $0 \leq t \leq t_{c}$.

b) Suppose that $|w(t)| \preceq w_{m}$ for all $t \geq 0$. Then, for any $\epsilon \in \mathbb{R}_{+}^{n}$, there exists a continuous function $\bar{t}_{f}(\epsilon, \cdot)$ : $\mathbb{R}^{n} \rightarrow \mathbb{R}_{+, 0}$ such that $|y(t)| \preceq R w_{m}+\tilde{R}\left|\xi_{0}\right|+|V| \epsilon$ for all $t \geq \bar{t}_{f}(\epsilon, y(0))$.

Proof of Claim 1. For a contradiction, suppose that $|x(t)|$ becomes unbounded. Note that $|x(t)| \preceq \theta_{0} \triangleq \theta(0)$ for $-\bar{\tau} \leq t \leq 0$ by $(3)$. Let $\delta \triangleq R \bar{w}+\tilde{R}|x(0)|+|x(0)|$, with $R$ and $\tilde{R}$ as in Lemma A.1, and let $\beta$ be given by Claim 4. Define

$$
t_{c} \triangleq \inf t, \quad \text { subject to } t \geq 0 \text { and }|x(t)| \npreceq \beta .
$$

Note that $\theta(t) \preceq \beta$ for all $0 \leq t \leq t_{c}$. From (2), then $|w(t)| \preceq w_{m} \triangleq F \beta+\bar{w}$ for all $0 \leq t \leq t_{c}$. Let $y(t) \triangleq$ $x(t)-x(0)$ and note that $y(0)=0$. Then, $y(t)$ verifies (A.1) with $\xi_{0}=x(0)$. Thus, applying Lemma A.1 a) it follows that $|y(t)| \preceq R w_{m}+\tilde{R}|x(0)|=R(F \beta+\bar{w})+$ $\tilde{R}|x(0)|$ for $0 \leq t \leq t_{c}$. Taking into account that $|x(t)| \preceq$ $|y(t)|+|x(0)|$ and using Claim 4, we obtain $|x(t)| \preceq$ $R(F \beta+\bar{w})+\tilde{R}|x(0)|+|x(0)|=R F \beta+\delta \prec \beta$, for $0 \leq t \leq$ $t_{c}$. From the continuity of $x(t)$, it then follows that there exists $\alpha \in \mathbb{R}_{+}$such that $|x(t)| \preceq \beta$ for $0 \leq t \leq t_{c}+\alpha$. This contradicts (A.2), concluding the proof.

Proof of Claim 2. By induction on $\ell$. By assumption, we have $|x(t)| \preceq \bar{x}$ for all $t \geq-\bar{\tau}$. From (2) and (3), then $|w(t)| \preceq \bar{F} \bar{x}+\bar{w}$, for all $t \geq 0$. Note that (A.1) coincides with $(5)$ when $\xi_{0}=0$ replacing $y(t)$ by $x(t)$. Thus, applying Lemma A.1 b) with $\xi_{0}=0$, then given $\gamma \in \mathbb{R}_{+}^{n}$, there exists a time instant $\bar{t}_{f}$ such that $|x(t)| \preceq$ $R(F \bar{x}+\bar{w})+|V| \gamma=T_{\gamma}^{0}(\bar{x})$ for all $t \geq \bar{t}_{f}$. The claim is then established for $\ell=0$ by setting $\bar{t}(0, \gamma)=\bar{t}_{f}$. Suppose next that the claim holds for some $\ell \in \mathbb{Z}_{+, 0}$, that is for any $\gamma \in \mathbb{R}_{+}^{n}$, there exists $\bar{t}(\ell, \gamma)$ such that $|x(t)| \preceq T_{\gamma}^{\ell+1}(\bar{x})$ for all $t \geq \bar{t}(\ell, \gamma)$. Then, by $(2), \theta(t) \preceq$ $T_{\gamma}^{\ell+1}(\bar{x})$ and hence, by $(3),|w(t)| \preceq F T_{\gamma}^{\ell+1}(\bar{x})+\bar{w}$, for $t \geq \bar{t}(\ell, \gamma)+\bar{\tau}$. Applying Lemma A.1 b) with $\xi_{0}=0$ and considering the fact that the system is time-invariant, then given $\gamma \in \mathbb{R}_{+}^{n}$, there exists $\tilde{t}_{f}$ such that $|x(t)| \preceq$ $R\left(F T_{\gamma}^{\ell+1}(\bar{x})+\bar{w}\right)+|V| \gamma=T_{\gamma}^{\ell+2}(\bar{x})$, for $t \geq \bar{t}(\ell, \gamma)+$ 
$\bar{\tau}+\tilde{t}_{f}$. The claim is then established for $\ell+1$ by setting $\bar{t}(\ell+1, \gamma)=\bar{t}(\ell, \gamma)+\bar{\tau}+\tilde{t}_{f}$. This concludes the proof. $\diamond$

Proof of Claim 3. Note that $\left|T_{\gamma}^{\ell+1}(\bar{x})-b\right| \preceq \mid T_{\gamma}^{\ell+1}(\bar{x})-$ $T^{\ell+1}(\bar{x})|+| T^{\ell+1}(\bar{x})-b \mid$. By $(7),(8)$, and since $\boldsymbol{\rho}(R F)<$ 1 , then $\lim _{\ell \rightarrow \infty} T^{\ell}(\bar{x})=b$. Also, since $\bar{x} \succeq 0$ and $T$ : $\mathbb{R}_{+, 0}^{n} \rightarrow \mathbb{R}_{+, 0}^{n}$, then $b \succeq 0$. Therefore, given $\epsilon \in \mathbb{R}_{+}^{n}$, we can select $\ell=\ell(\epsilon)$ such that $\left|T^{\ell+1}(\bar{x})-b\right| \prec \epsilon / 2$. From (8), it follows straightforwardly that, for the selected value of $\ell$, we may select $\gamma=\gamma(\epsilon) \in \mathbb{R}_{+}^{n}$ small enough so that $\left|T_{\gamma}^{\ell+1}(\bar{x})-T^{\ell+1}(\bar{x})\right| \prec \epsilon / 2$. Then, $\left|T_{\gamma}^{\ell+1}(\bar{x})-b\right| \prec \epsilon$. Since $b \succeq 0$ and $T_{\gamma}^{0}: \mathbb{R}_{+, 0}^{n} \rightarrow \mathbb{R}_{+, 0}^{n}$ for any $\gamma \in \mathbb{R}_{+, 0}^{n}$, then $T_{\gamma}^{\ell+1}(\bar{x})=\left|T_{\gamma}^{\ell+1}(\bar{x})-b+b\right| \preceq\left|T_{\gamma}^{\ell+1}(\bar{x})-b\right|+b \prec b+\epsilon$, establishing the claim.

\section{B Proof of Theorem 4.2}

From Sinswat and Fallside (1977), we know that a necessary and sufficient condition for a matrix $K_{c} \in \mathbb{R}^{m \times n}$ to exist such that the pair $(\lambda, \tilde{e}), \lambda \in \mathbb{C}, \tilde{e} \in \mathbb{C}^{n}$ is an eigenvalue-eigenvector pair of $A_{c}+B_{c} K_{c}$, i.e., such that $(\lambda, \tilde{e})$ is assignable by state feedback, is that

$$
\Gamma \tilde{e}=0, \quad \text { where } \quad \Gamma \triangleq\left(\mathrm{I}-B_{c} B_{c}^{\dagger}\right)\left(A_{c}-\lambda \mathrm{I}\right) .
$$

When the system is in controller canonical form, the matrix $\Gamma$ in (B.1) can be shown to have the simple form

$$
\Gamma=\operatorname{diag}\left(\Gamma_{1}, \ldots, \Gamma_{m}\right), \Gamma_{i} \triangleq\left[\begin{array}{ccccc}
-\lambda & 1 & 0 & \cdots & 0 \\
0 & -\lambda & 1 & \cdots & 0 \\
\vdots & \ddots & \ddots & \ddots & \vdots \\
0 & \cdots & 0 & -\lambda & 1 \\
0 & \cdots & 0 & 0 & 0
\end{array}\right] \in \mathbb{R}^{d_{i} \times d_{i}}
$$

Using (B.1)-(B.2), it follows that the pair $(\lambda, \tilde{e})$, where $\lambda \in \mathbb{C}, \lambda \neq 0, \tilde{e} \triangleq\left[\begin{array}{cccc}\tilde{e}_{1} & \tilde{e}_{2} & \ldots & \tilde{e}_{n}\end{array}\right]^{T} \in \mathbb{C}^{n}$ is an assignable eigenvalue-eigenvector pair if and only if

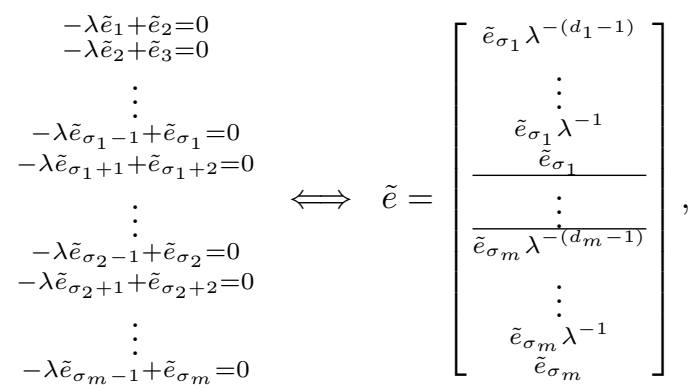

where we have defined $\sigma_{i} \triangleq \sum_{j=1}^{i} d_{j}$ for $i=1, \ldots, m$ (note that $\sigma_{m}=n$ ). The above equations imply that if $e_{1} \triangleq \tilde{e}_{\sigma_{1}}, e_{2} \triangleq \tilde{e}_{\sigma_{2}}, \ldots, e_{m} \triangleq \tilde{e}_{\sigma_{m}}$ are freely chosen then the remaining components of $\tilde{e}$ are fixed. Since the columns of $V$ in (12) have the form of $\tilde{e}$ in (B.3), we conclude that $V$ is an assignable eigenvector matrix. Finally, also according to Sinswat and Fallside (1977), the feedback matrix required to assign the eigenvalueeigenvector pairs selected is $K_{c}$ as in (13).

\section{References}

B.R. Barmish, I.R. Petersen, and A. Feuer. Linear ultimate boundedness control of uncertain dynamical systems. Automatica, 19(5):523-532, 1983.

M.L. Brockman and M. Corless. Quadratic boundedness of nominally linear systems. International Journal of Control, 71(6):1105-1117, 1998.

Y.-Y. Cao and Y.-X. Sun. Robust stabilization of uncertain systems with time-varying multistate delay. IEEE Trans. on Automat. Contr., 43(10):1484-1488, 1998.

H. Haimovich. Quantisation issues in feedback control. PhD thesis, School of Electrical Engineering and Computer Science, The University of Newcastle, Australia, March 2006. URL http: //usuarios.fceia.unr.edu.ar/ haimo.

R. A. Horn and C. R. Johnson. Matrix Analysis. Cambridge University Press, Cambridge, UK, 1985.

H. Khalil. Nonlinear Systems. Prentice-Hall, New Jersey, 3rd edition, 2002.

E. Kofman. Non-conservative ultimate bound estimation in LTI perturbed systems. Automatica, 41(10): 1835-1838, October 2005.

E. Kofman, H. Haimovich, and M. Seron. A systematic method to obtain ultimate bounds for perturbed systems. International Journal of Control, 80(2):167178, February 2007a.

E. Kofman, M.M. Seron, and H. Haimovich. Robust control design with guaranteed state ultimate bound. In 3rd International Conference on Integrated Modeling and Analysis in Applied Control and Automation IMAACA, Buenos Aires, Argentina, 2007b. URL www . ee.newcastle.edu.au/reports/EE07011.pdf.

D. Luenberger. Canonical forms for linear multivariable systems. IEEE Trans. Automat. Contr., 12:290-293, 1967.

Said Oucheriah. Robust tracking and model following of uncertain dynamic delay systems by memoryless linear controllers. IEEE Trans. Automat. Contr., 44 (7):1473-1477, 1999.

W. E. Schmitendorf and B. R. Barmish. Robust asymptotic tracking for linear systems with unknown parameters. Automatica, 22:355-360, 1986.

V. Sinswat and F. Fallside. Eigenvalue/eigenvector assignment by state-feedback. International Journal of Control, 26(3):389-403, 1977.

H. Trinh and M. Aldeen. Output tracking for linear uncertain time-delay systems. IEE Proceedings, Control Theory and Applications, 143(6):481-488, 1996.

W. A. Wolovich. Linear multivariable systems. SpringerVerlag, New York, 1974. 